\title{
A Case of Bilateral Suppurative Arthritis of Hip Joint with Concomitant Pyomyositis
}

\author{
Halil Atmaca ${ }^{1}$, Kaya Memişoğlu², Zeki Yumuk ${ }^{3}$ Adem Aydın \\ 1Department of Orthopaedics and Traumatology, Midyat State Hospital, Mardin, Turkey \\ ${ }^{2}$ Department of Orthopaedics and Traumatology, Faculty of Medicine, Kocaeli University, Kocaeli, Turkey \\ ${ }^{3}$ Department of Microbiology, Faculty of Medicine, Kocaeli University, Kocaeli, Turkey \\ ${ }^{4}$ Department of Orthopaedics and Traumatology, Izmit Seka State Hospital, Kocaeli, Turkey
}

Hip pain in a child is a common presentation and may pose a diagnostic challenge. Septic arthritis, transient synovitis, Perthes disease, and slipped capital femoral ephiphysis are the most important causes of hip pain among the children aged 3-10 years (1). Fracture, inflammatory arthropathy and tumors are other causes of hip irritability. Diagnosis of pyomyositis involving muscles around the hip may be problematic because of its rarity, its indolent presentation, and the similarity of its clinical features with those of septic arthritis. The differential diagnosis can be made on the basis of the history, physical examination and imaging studies. The problem often left to the clinician is to choose between septic arthritis and transient synovitis, the two most probable etiologies (2).

A 7-year-old boy presented with a five-day history of pain in both hips. There was no history of trauma or any other apparent reason for the pain. He had been treated by a physician with oral cephalosporins for five days prior to his presentation. His family history was not contributory. The patient had a continuous temperature of $38.5^{\circ} \mathrm{C}$ and was unable to extend, abduct or rotate his hips because of the pain and kept them in $80^{\circ}$ flexion. He had a white blood cell (WBC) count of $13.900 / \mu \mathrm{L}$, an erythrocyte sedimentation rate (ESR) of $82 \mathrm{~mm} /$ hour and a C-reactive protein (CRP) of $5.51 \mathrm{mg} /$ dL. Plain radiographs of the pelvis demonstrated no abnormalites. Ultrasound examination showed fluid collection in both hip joints. Magnetic resonance imaging (MRI) revealed pyomyositis of the left obturator externus and pectineus muscles with bilateral hip joint effusion, suggesting septic arthritis (Figure 1). Bilateral hip joint aspirate revealed a straw-colored fluid containing $18.356 \mathrm{WBCs} / \mu \mathrm{L}$ (80\% neutrophils, $15 \%$ lymphocytes, and $5 \%$ monocytes). Glucose concentration of the fluid was $<20 \mathrm{mg} / \mathrm{dL}$. The pathology of the tissue specimen revealed an exudate full of neutrophils and fibrin clusters. The Gram smear showed gram (+) stained cocci but no bacterial growth occurred in joint fluid cultures.

Surgical drainage was performed through a posterior approach for both hips. There was exudative high viscosity joint liquid in both hips. Empiric intravenous clindamycin and cefazoline were started and continued for six weeks. Blood and

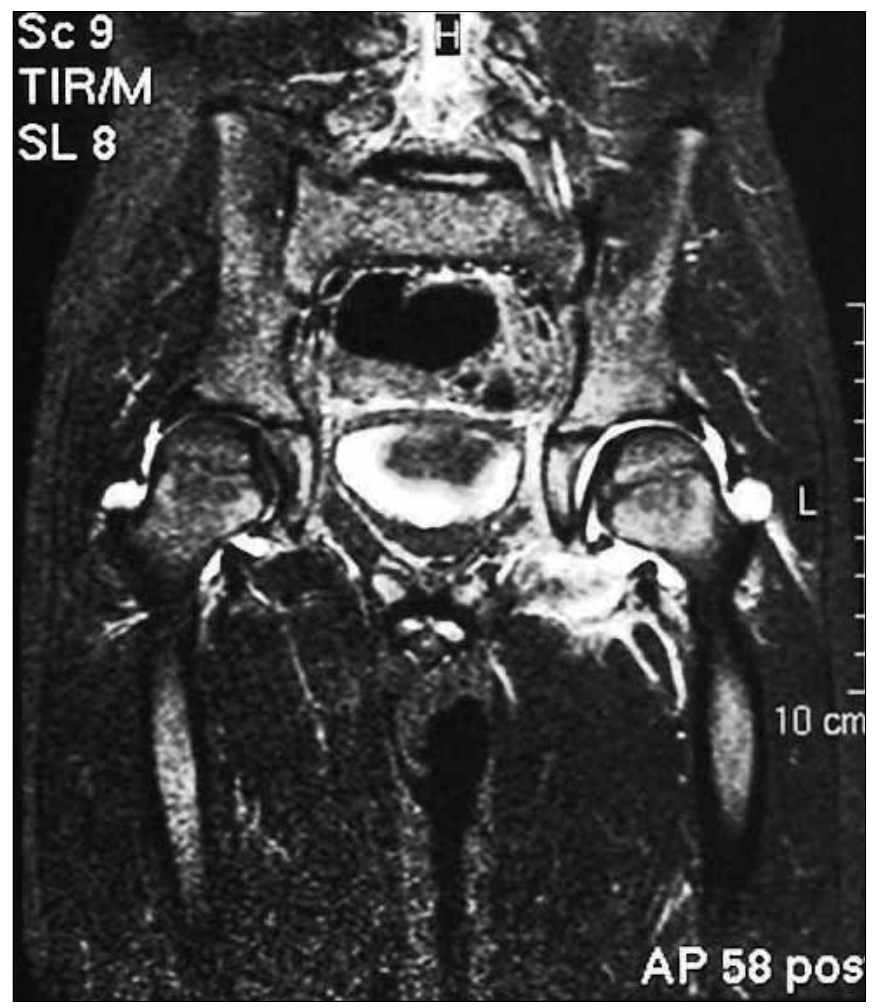

Figure 1. MRI of the pelvis reveals pyomyositis of the left obturator externus and pectineus muscles with bilateral hip joint effusion suggesting septic arthritis

pus cultures were positive for Staphylococcus aureus sensitive to clindamycin. Within days, the patient's pain has ceased, together with the normalization of ESR and CRP concentrations. Four months after his discharge, he was asymptomatic and had full range of motion in both hips.

Prompt and meticulous differential diagnosis of this patient, which included septic arthritis, transient synovitis, and pyomyositis as the most frequently encountered causes of hip pain, was critical because of the vastly different treatments and potential for sequelae of each clinical entity $(3,4)$. Eich et 
al. (5) concluded that a rectal temperature of $\geq 38^{\circ} \mathrm{C}$, an erythrocyte sedimentation rate of $\geq 20 \mathrm{~mm} /$ hour, and a C-reactive protein concentration of $\geq 20 \mathrm{mg} / \mathrm{dL}$ ( $200 \mathrm{mg} / \mathrm{L}$ ) were the most important characteristics for differentiation of septic arthritis and transient synovitis. If two of these parameters were positive and ultrasonography showed a hip effusion, there was a sensitivity of $100 \%$ and a specificity of $89 \%$ for septic arthritis. Whereas the normal-to-slightly high WBC count of our patient was not in favour of an infection, a body temperature of $38.5^{\circ} \mathrm{C}$, neutrophil predominance in the peripheral smear, and elevated acute phase reactants might be suggestive of septic arthritis, although these laboratory parameters are very well known to lack a definitive diagnostic value. Furthermore, bilaterality of the symptoms made the diagnosis extremely difficult as bilateral septic arthritis is a rare condition (6).

Luhmann indicated in their review that a previous antibiotic treatment may camouflage the clinical picture of septic arthritis (7), which should be the case for our patient, who had been on cephalosporins for a few days. Even if the patient were "antibiotic-free", the chances that we could grow the causative organism would not be expected to be more than $30 \%$ (8). Thus, although the causative organism of the septic arthritis could not be identified, the clinical and laboratory findings pointed to the diagnosis without any suspicion. In addition to the unexpected finding of bilateral septic arthritis, another rarity was the association of pyomyositis, which was detected in the MRI. The most common etiologic organism is Staphylococcus aureus, but Streptococcus, Escherichia coli, and Enterococcus have also been reported (9). The pathophysiology of the pyomyositis is unclear and there is no clear evidence to the support trauma of the affected muscle with transient bacteremia theory, as in our patient's history (10). In addition to symptoms and signs of osteomyelitis, cellulitis and malignant tumors; pyomyositis may manifest itself mimicking septic arthritis, which might have accentuated the clinical picture of our patient. The use of MRI in such cases is the mainstay of diagnosis.

What we have learned from this case is that septic arthritis might be bilateral and that bilateral septic arthritis and pyomyositis should be considered in the differential diagnosis of children with hip pain.

\section{References}

1. Shah SS. Abnormal gait in a child with fever: diagnosing septic arthritis of the hip. Pediatr Emerg Care 2005;21:336-41. [CrossRef]

2. Luhmann SJ, Jones A, Schootman M, Gordon E, Schoenecker PL, Luhmann JD. Differentiation between septic arthritis and transient synovitis of the hip in children with clinical prediction algorithms. J Bone Joint Surg 2004;86:956-62.

3. Fabry G, Meire E. Septic arthritis of the hip in children: poor results after late and inadequate treatment. J Pediatr Orthop 1983;3:461-6. [CrossRef]

4. Wopperer JM, White JJ, Gillespie R, Obletz BE. Long term follow-up of infantile hip sepsis. J Pediatric Orthop 1988;8:322-5. [CrossRef]

5. Eich GF, Superti-Furga A, Umbricht FS, Willi UV. The painful hip: evaluation of criteria for clinical decision-making. Eur J Pediat 1999;159:923-8. [CrossRef]

6. Ibn Yacoub Y, Amine B, Hajjaj-Hassouni N. Bilateral septic arthritis of the hip with osteitis and psoas abcess in a 17-year-old adolescent. J Pediatr Orthop B 2011;20:238-41. [CrossRef]

7. Luhmann JD, Luhmann SJ. Etiology of septic arthritis in children: an update for the 1990s. Ped Emerg Care 1999;15:40-2. [CrossRef]

8. Lyon RM, Evanich JD. Culture-negative septic arthritis in children. J Pediatric Orthop 1999;19:655-9. [CrossRef]

9. Wong-Chung J, Bagali M, Kaneker S. Physical signs in pyomyositis presenting as a painful hip in children: a case report and review of literature. J Pediatr Orthop B 2004;13:211-3. [CrossRef]

10. Bickels J, Ben-Sira L, Kessler A, Wientroub S. Primary pyomyositis. J Bone Joint Surg Am 2002;84:2277-86. 